To appear in the Journal of Modern Optics

Vol. 00, No. 00, 00 Month 20XX, 1-17

\title{
Coherence properties of the radiation from FLASH
}

\author{
E.A. Schneidmiller, M.V. Yurkov, DESY, Hamburg, Germany \\ (Received 00 Month 20XX; final version received 00 Month 20XX)
}

\begin{abstract}
FLASH is the first free electron laser user facility operating in the vacuum ultraviolet and soft $\mathrm{x}$ ray wavelength range. Many user experiments require knowledge of the spatial and temporal coherence properties of the radiation. In this paper we present an analysis of the coherence properties of the radiation for the fundamental and for the higher odd frequency harmonics. We show that temporal and spatial coherence reach maximum close to the FEL saturation but may degrade significantly in the postsaturation regime. We also find that the pointing stability of short FEL pulses is limited due to the fact that non-azimuthal FEL eigenmodes are not sufficiently suppressed. We discuss possible ways for improving the degree of transverse coherence and the pointing stability.
\end{abstract}

\section{Introduction}

FLASH (Free electron LASer in Hamburg) operates in the vacuum-ultraviolet and soft X-ray range between approximately $45 \mathrm{~nm}$ and $4.2 \mathrm{~nm}$ wavelength. This facility originated from the TESLA Test Facility (TTF) project which was built to test the technology for the linear collider TESLA (TeV Energy Superconducting Linear Collider) [1 3]. At the same time the project of the TTF free electron laser (TTF FEL) has been launched aiming a minimum radiation wavelength of $6 \mathrm{~nm}$ [4]. The first stage of the project successfully generated VUV light in the year 2000 [5]. High power (a few GW) and ultrashort (a few $10 \mathrm{fs}$ ) radiation pulses have been used in pioneer user experiments [6 9]. It has been decided later on to transform the TTF FEL to the dedicated FEL user facility which is in operation since 2004 under the name of FLASH 10 16]. FLASH free electron laser is driven by $1.25 \mathrm{GeV}$ superconducting linear accelerator. Five scientific instruments have been in use since the commissioning of the facility in 2004. Second stage, FLASH2 is under commissioning now. First lasing at FLASH2 has been obtained in August, 2014 [15, 16]. FLASH facility is also used for the development and testing of technology for the European XFEL and for the International Linear Collider (ILC) [17-20].

With the present undulator (period $2.73 \mathrm{~cm}$, peak field $0.486 \mathrm{~T}$ ) the minimum wavelength of 4.2 $\mathrm{nm}$ is determined by the maximum electron beam energy of approximately $1.25 \mathrm{GeV}$. There is a tendency for users at FLASH to extend wavelength range to shorter wavelengths. The first target is the so-called water window, i.e. the range between the K-Absorption edges of carbon and oxygen at $4.38 \mathrm{~nm}$ and $2.34 \mathrm{~nm}$, respectively. Currently a minimum wavelength of FLASH is just below the carbon edge. Another range of interest refers to the edges of magnetic elements which spans below water window. Higher odd harmonics of SASE radiation can be used to generate radiation at such short wavelengths. Pioneer experiment for studying magnetic materials using FEL radiation has been performed at FLASH at $1.6 \mathrm{~nm}$ wavelength, the 5 th harmonic of the fundamental at 8 $\mathrm{nm}$ [21]. Many user's experiments rely on coherent properties of the radiation, both temporal and spatial. This relates not only to the fundamental harmonic, but to the higher odd harmonics as well [22, 23].

FLASH is single pass free electron laser starting from the shot noise in the electron beam [24 26]. This FEL amplifier configuration is frequently named as SASE FEL (Self Amplified Spontaneous Emission FEL [27]). Previous studies have shown that coherence properties of the radiation from 
SASE FEL strongly evolve during the amplification process [28 32]. At the initial stage of amplification the spatial coherence is poor, and the radiation consists of a large number of transverse modes [32 40]. Longitudinal coherence is poor as well [41-43]. In the exponential stage of amplification the transverse modes with higher gain dominate over modes with lower gain when the undulator length progresses. This feature is also known as the mode competition process. Longitudinal coherence is also improving in the high gain linear regime [43 45]. Mode selection process stops at the onset of the nonlinear regime, and maximum values of the degree of the transverse coherence and of the coherence time are reached at this point. Undulator length to saturation is in the range from about nine (hard x-ray SASE FELs) to eleven (visible range SASE FELs) field gain lengths [28]. Situation with the transverse coherence is favorable when the relative separation of the field gain between fundamental and higher modes exceeds 25-30\%. In this case the maximum degree of transverse coherence can exceed the value of $90 \%$ [28, 32]. Further development of the amplification process in the nonlinear stage leads to visible degradation of the coherence properties.

Separation of the gain of the FEL radiation modes mainly depends on the value of the diffraction parameter. Increase of the value of the diffraction parameter results in less relative separation of the gain of the modes. In this case we deal with the mode degeneration [34, 37]. Since the number of gain lengths to saturation is limited, the contribution of the higher spatial modes to the total power grows with the value of the diffraction parameter, and the transverse coherence degrade. Parameter range of large diffraction parameter values is typical for SASE FELs operating in the hard x-ray wavelength range [18, 46 49]. It is also worth noticing that a spread of longitudinal velocities (due to energy spread and emittance) helps to suppress high order modes thus improving transverse coherence properties. This consideration suggests that a tight focusing of the electron beam in the undulator can be important for reaching a good coherence due to a reduction of the diffraction parameter and an increase of the velocity spread.

In this paper we perform thorough analysis of the coherence properties of the radiation from FLASH free electron laser. We found that the degree of transverse coherence of the radiation from FLASH is visible less than unity in the post-saturation regime. Moreover, we find that the pointing stability of the FEL beam suffers from not sufficient mode selection of higher spatial radiation modes which happens due to large values of the diffraction parameter. Our analysis shows that operation with a stronger focusing of the electron beam and a lower peak current would allow to improve degree of transverse coherence and the pointing stability.

\section{Analysis of the radiation modes}

We consider axisymmetric model of the electron beam. It is assumed that transverse distribution function of the electron beam is Gaussian, so rms transverse size of matched beam is $\sigma=\sqrt{\epsilon \beta}$, where $\epsilon$ is rms beam emittance and $\beta$ is the beta-function. In the framework of the three-dimensional theory the operation of a short-wavelength FEL amplifier is described by the following parameters: the diffraction parameter $B$, the energy spread parameter $\hat{\Lambda}_{\mathrm{T}}^{2}$, the betatron motion parameter $\hat{k}_{\beta}$ and detuning parameter $\hat{C}[36,37]$ :

$$
\begin{aligned}
B & =2 \Gamma \sigma^{2} \omega / c, & \hat{C} & =C / \Gamma, \\
\hat{k}_{\beta} & =1 /(\beta \Gamma), & \hat{\Lambda}_{\mathrm{T}}^{2} & =\left(\sigma_{\mathrm{E}} / E\right)^{2} / \rho^{2},
\end{aligned}
$$

where $E=\gamma m c^{2}$ is the energy of electron, $\gamma$ is relativistic factor, $\Gamma=\left[I \omega^{2} \theta_{\mathrm{s}}^{2} A_{\mathrm{JJ}}^{2} /\left(I_{\mathrm{A}} c^{2} \gamma_{\mathrm{z}}^{2} \gamma\right)\right]^{1 / 2}$ is the gain parameter, $\rho=c \gamma_{\mathrm{z}}^{2} \Gamma / \omega$ is the efficiency parameter, and $C=2 \pi / \lambda_{\mathrm{w}}-\omega /\left(2 c \gamma_{z}^{2}\right)$ is the detuning of the electron with the nominal energy $\mathcal{E}_{0}$. Note that the efficiency parameter $\rho$ entering equations of three dimensional theory relates to the one-dimensional parameter $\rho_{1 \mathrm{D}}$ as $\rho_{1 \mathrm{D}}=\rho / B^{1 / 3}$ [37, 50]. The following notations are used here: $I$ is the beam current, $\omega=2 \pi c / \lambda$ 
is the frequency of the electromagnetic wave, $\lambda_{\mathrm{w}}$ is undulator period, $\theta_{\mathrm{s}}=K / \gamma, K$ is the rms undulator parameter, $\gamma_{z}^{-2}=\gamma^{-2}+\theta_{\mathrm{s}}^{2}, I_{\mathrm{A}}=m c^{3} / e=17 \mathrm{kA}$ is the Alfven current, $A_{\mathrm{JJ}}=1$ for helical undulator and $A_{\mathrm{JJ}}=J_{0}\left(K^{2} / 2\left(1+K^{2}\right)\right)-J_{1}\left(K^{2} / 2\left(1+K^{2}\right)\right)$ for planar undulator. $J_{0}$ and $J_{1}$ are the Bessel functions of the first kind. The energy spread is assumed to be Gaussian with rms deviation $\sigma_{\mathrm{E}}$.

Amplification process in SASE FEL starts from the shot noise in the electron beam. At the initial stage of amplification coherence properties are poor, and the radiation consists of a large number of transverse and longitudinal modes 32 40]:

$$
\tilde{E}=\sum_{m, n} \int \mathrm{d} \omega A_{m n}(\omega, z) \Phi_{m n}(r, \omega) \exp \left[\Lambda_{m n}(\omega) z+i m \phi+i \omega(z / c-t)\right]
$$

Each mode is characterized by the eigenvalue $\Lambda_{m n}(\omega)$ and the field distribution eigenfunction $\Phi_{m n}(r, \omega)$. Real part of the eigenvalue $\operatorname{Re}\left(\Lambda_{m n}(\omega)\right)$ is referred as the field gain. The field gain length is $L_{g}=1 / \operatorname{Re}\left(\Lambda_{m n}(\omega)\right)$. Eigenvalues and eigenfunctions are the solutions of the eigenvalue equation [35, 36]. Each eigenvalue has a maximum at a certain frequency (or, at a certain detuning),

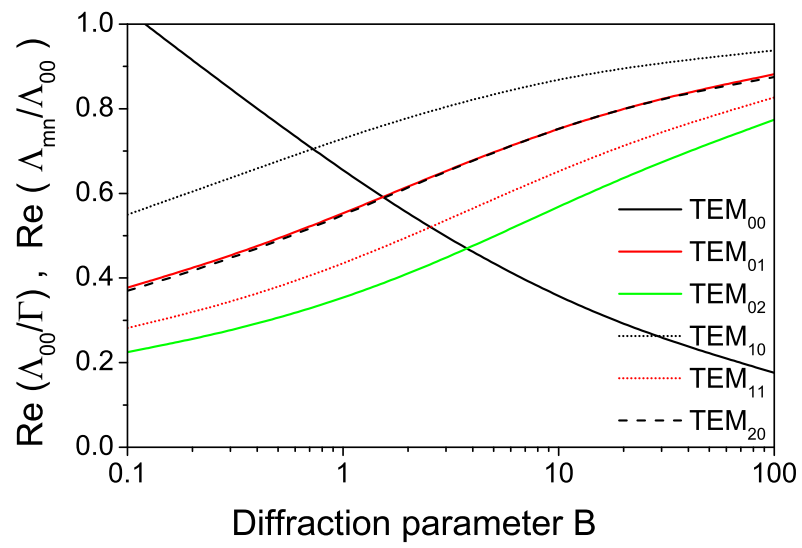

Figure 1. Ratio of the maximum gain of the higher modes to the maximum gain of the fundamental mode $\operatorname{Re}\left(\Lambda_{m n}\right) / \operatorname{Re}\left(\Lambda_{00}\right)$ versus diffraction parameter $B$. The energy spread parameter is $\hat{\Lambda}_{\mathrm{T}}^{2} \rightarrow 0$, and the betatron motion parameter is $\hat{k}_{\beta} \rightarrow 0$. Color codes refer to the radial index of the mode: 0 - black, 1 - red, 2 - green. Line type codes refer to the azimuthal index of the mode: 0 - solid line, 1 - dotted line, 2 - dashed line. Black solid line shows the gain of the fundamental $\operatorname{mode} \operatorname{Re}\left(\Lambda_{00}\right) / \Gamma$.
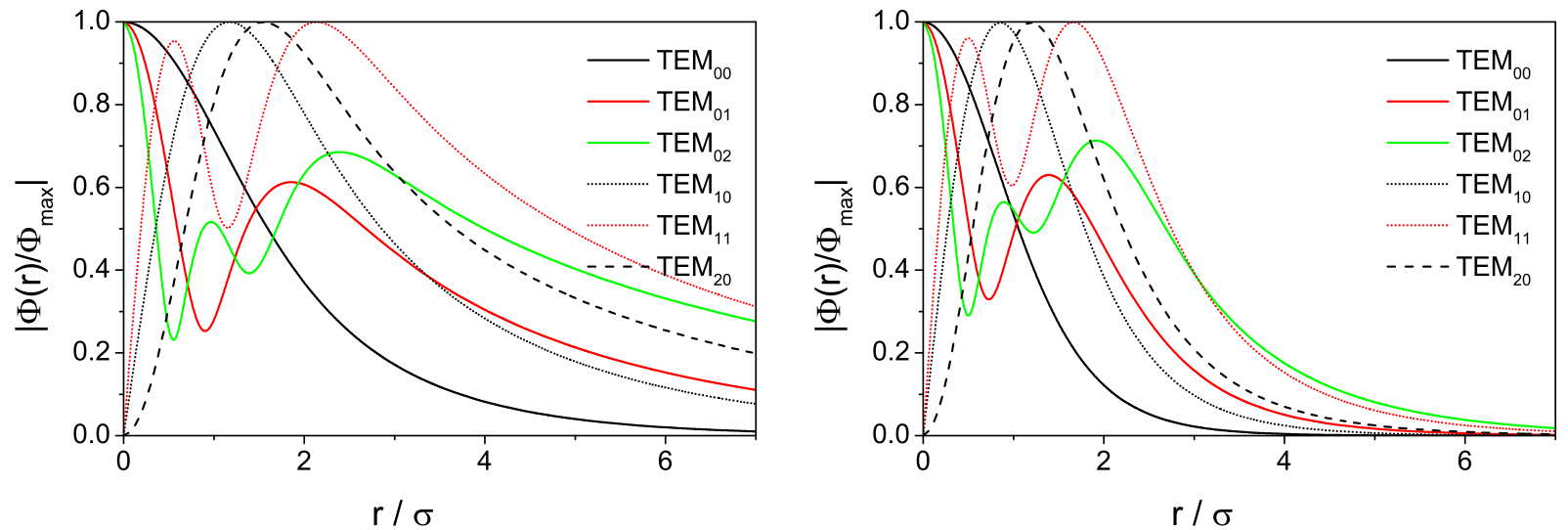

Figure 2. Amplitude of the eigenfunctions of the FEL radiation modes, $\left|\Phi_{m n}(r)\right| /\left|\Phi_{\max }\right|$. Left and right plot correspond to the diffraction parameter $B=1$ and $B=10$, respectively. The detuning corresponds to the maximum of the gain. The energy spread parameter is $\hat{\Lambda}_{\mathrm{T}}^{2} \rightarrow 0$, and the betatron motion parameter is $\hat{k}_{\beta} \rightarrow 0$. Color codes refer to the radial index of the mode: 0 - black, 1 - red, 2 - green. Line type codes refer to the azimuthal index of the mode: 0 - solid line, 1 - dotted line, 2 - dashed line. 


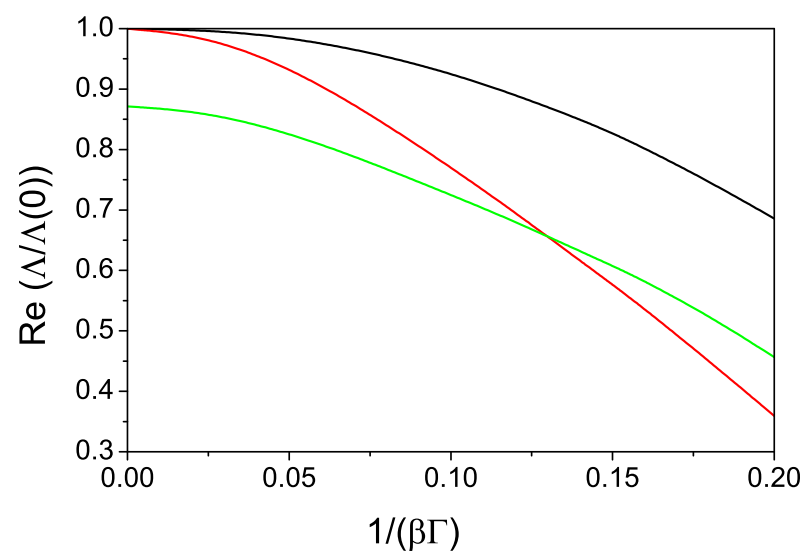

Figure 3. Dependence of the gain of $\mathrm{TEM}_{00}$ mode (black curve) and $\mathrm{TEM}_{10}$ mode (red curve) on the betatron motion parameter $\hat{k}_{\beta}=1 /(\beta \Gamma)$. The values are normalized to those at $\hat{k}_{\beta} \rightarrow 0$. Green curve shows the ratio of the gain of TEM 10 mode to the gain of $\mathrm{TEM}_{00}$ mode. The diffraction parameter is $B=10$. The energy spread parameter is $\hat{\Lambda}_{\mathrm{T}}^{2} \rightarrow 0$.

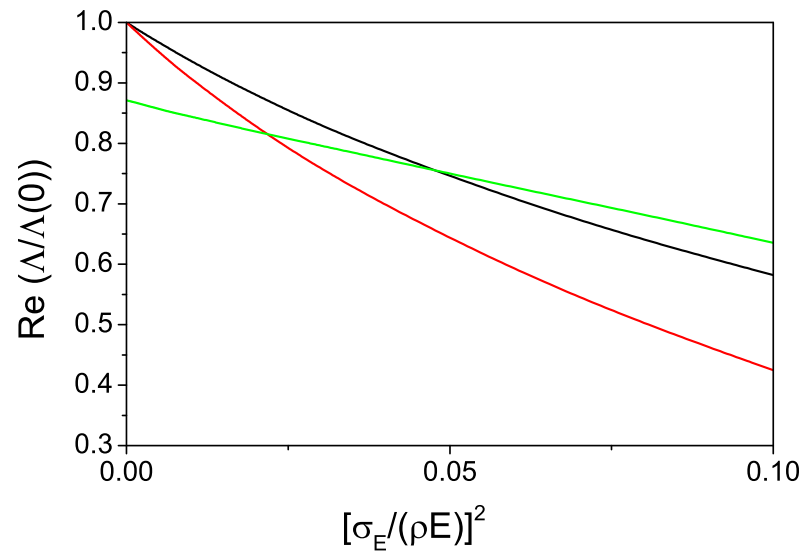

Figure 4. Dependence of the gain of TEM 00 mode (black curve) and TEM 10 mode (red curve) on the energy spread parameter $\hat{\Lambda}_{\mathrm{T}}^{2}$. The values are normalized to those at $\hat{\Lambda}_{\mathrm{T}}^{2} \rightarrow 0$. Green curve shows the ratio of the gain of TEM 10 mode to the gain of $\mathrm{TEM}_{00}$ mode. The diffraction parameter is $B=10$. The betatron oscillation parameter is $\hat{k}_{\beta} \rightarrow 0$.

so that the detunig for each mode is chosen automatically in the case of a SASE FEL (in contrast with seeded FELs where the detuning can be set to any value). Thus, we will in fact deal with the three dimensionless parameters: $B, \hat{k}_{\beta}$, and $\hat{\Lambda}_{\mathrm{T}}^{2}$.

Let us look closer at the properties of the radiation modes. The gains for several modes is depicted in Fig. 6 as functions of the diffraction parameter. The values for the gain correspond to the maximum of the scan over the detuning parameter $\hat{C}$. The curve for $\mathrm{TEM}_{00}$ mode shows the values of normalized gain $\operatorname{Re}\left(\Lambda_{00} / \Gamma\right)$. Curves for the higher spatial modes present the ratio of the gain of the mode to the gain of the fundamental mode, $\operatorname{Re}\left(\Lambda_{m n} / \Lambda_{00}\right)$. Sorting of the modes by the gain results in the following ranking: $\mathrm{TEM}_{00}, \mathrm{TEM}_{10}, \mathrm{TEM}_{01}, \mathrm{TEM}_{20}, \mathrm{TEM}_{11}, \mathrm{TEM}_{02}$. The gain of the fundamental $\mathrm{TEM}_{00}$ mode is always above the gain of higher order spatial modes. The difference in the gain between the fundamental $\mathrm{TEM}_{00}$ mode and higher spatial modes is pronouncing for small values of the diffraction parameter $B \lesssim 1$. The gain of higher spatial modes approaches asymptotically the gain of the fundamental mode for large values of the diffraction parameter. In other words, the effect of the mode degeneration takes place. Its origin can be understood with the qualitative analysis of the eigenfunctions (distribution of the radiation field in the near zone). Figure 2 shows eigenfunctions of the FEL radiation modes for two values of the diffraction parameter, $B=1$ and $B=10$. We observe that for small values of the diffraction parameter the field of the higher spatial modes spans far away from the core of the electron beam while the fundamental $\mathrm{TEM}_{00}$ mode is more confined. This feature provides higher coupling factor of the 
radiation with the electron beam and higher gain. For large values of the diffraction parameter all radiation modes shrink to the beam axis which results in equalizing of coupling factors and of the gain. Asymptotically, the eigenvalues of all modes tends to the one dimensional asymptote as [30]:

$$
\Lambda_{m n} / \Gamma \simeq \frac{\sqrt{3}+\mathrm{i}}{2 B^{1 / 3}}-\frac{(1+\mathrm{i} \sqrt{3})(1+n+2 m)}{3 \sqrt{2} B^{2 / 3}}
$$

For a SASE FEL, the undulator length to saturation is in the range from about nine (hard x-ray range) to eleven (visible range) field gain lengths [28, 29, 31]. The mode selection process stops at the onset of nonlinear regime, about two field gain length before the saturation. Let us make simple estimation for the value of the diffraction parameter $B=10$ and cold electron beam, $\hat{\Lambda}_{\mathrm{T}}^{2} \rightarrow 0$, and $\hat{k}_{\beta} \rightarrow 0$. We get from Fig. 6 that the ratio of the gain $\operatorname{Re}\left(\Lambda_{10} / \Lambda_{00}\right)$ is equal to 0.87 . With an assumption of similar values of coupling factors, we find that the ratio of the field amplitudes at the onset of the nonlinear regime is about of factor of 3 only. An estimate for the contribution of the higher spatial modes to the total power is about $10 \%$. Another numerical example for $B=1$ gives the ratio $\operatorname{Re}\left(\Lambda_{10} / \Lambda_{00}\right)=0.73$, and the ratio of field amplitudes exceeds a factor of 10 . Thus, an excellent transverse coherence of the radiation is not expected for SASE FEL with diffraction parameter $B \gtrsim 10$ and a small velocity spread in the electron beam.

Longitudinal velocity spread due to the energy spread and emittance serves as a tool for selective suppression of the gain of the higher spatial modes [34, 37]. Figures 3 and 4 show the dependence of the gain of $\mathrm{TEM}_{00}$ and $\mathrm{TEM}_{10}$ modes on the betatron motion parameter and the energy spread parameter. We see that with the fixed value of the diffraction parameter, the mode degeneration effect can be relaxed at the price of the gain reduction.

The betatron motion can influence the gain of different modes (and, therefore, transverse coherence properties) via two different mechanisms. First, the particles move across the beam thus transferring the information between different points in the beam cross-section. Second, as it was already mentioned, there is a spread of longitudinal velocities that has a similar effect as the energy spread (and it is usually more important than the first one). One can introduce a combination of parameters $B$ and $\hat{k}_{\beta}$ that can to some extent be similar with the energy spread parameter:

$$
\left(\hat{\Lambda}_{\mathrm{T}}^{2}\right)_{e f f}=B^{2} \hat{k}_{\beta}^{4}
$$

Finally, let us note that the situation with transverse coherence is favorable when relative separation of the gain between the fundamental and higher spatial modes is more than 25-30\%. In this case the degree of transverse coherence can reach values above $90 \%$ in the end of the high gain linear regime [30, 32]. Further development of the amplification process in the nonlinear stage leads to a significant degradation of the spatial and of the temporal coherence [28, 29, 31].

\section{Parameter space of FLASH}

In the present experimental situation, many parameters of the electron beam at FLASH depend on practical tuning of the machine. Analysis of measurements and numerical simulations shows that depending on tuning of the machine emittance may change from about 1 to about $1.5 \mathrm{~mm}-\mathrm{mrad}$. Tuning at small charges may allow to reach smaller values of the emittance down to $0.5 \mathrm{~mm}$-mrad. Peak current may change in the range from $1 \mathrm{kA}$ to $2 \mathrm{kA}$ depending on the tuning of the beam formation system. An estimate for the local energy spread is $\sigma_{\mathrm{E}}[\mathrm{MeV}] \simeq 0.1 \times I[\mathrm{kA}]$. The average beta function in the undulator is about 10 meters.

Let us choose the reference working point with the radiation wavelength $8 \mathrm{~nm}$, rms normalized emittance $1 \mathrm{~mm}$-mrad and beam current $1.5 \mathrm{kA}$. Parameters of the problem for this reference point 

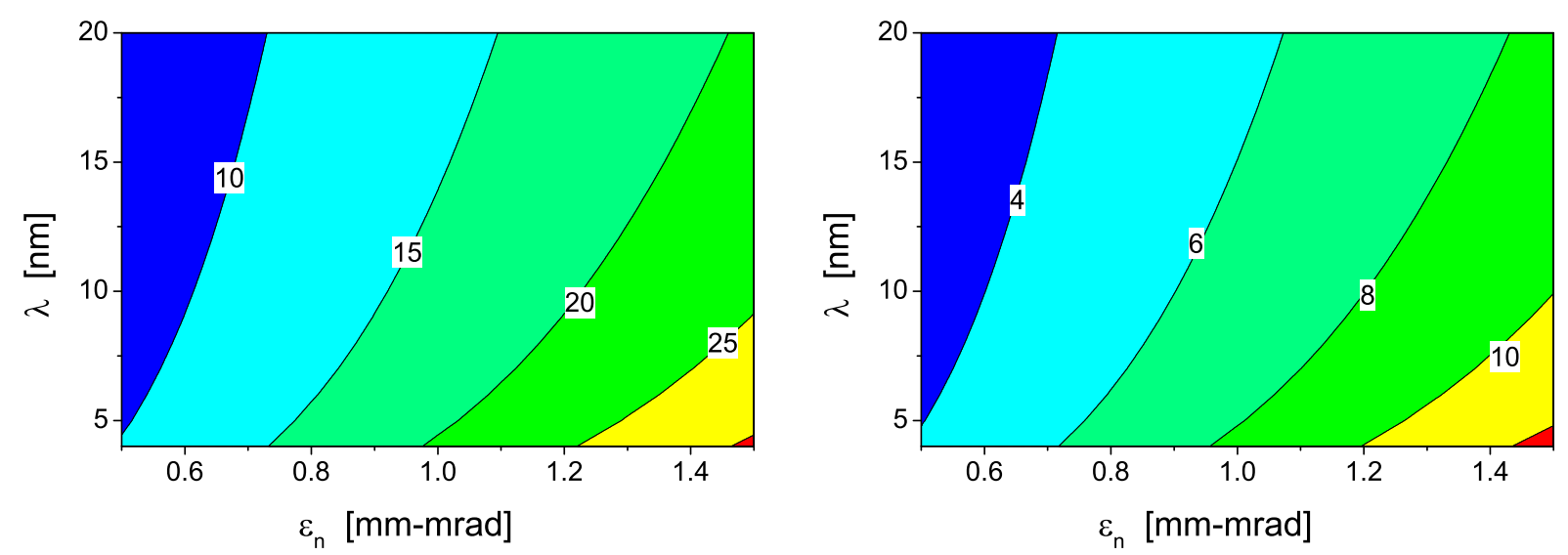

Figure 5. Contour plot for the value of the diffraction parameter $B$ versus normalized emittance and radiation wavelength. Left plot: beam current is $1.5 \mathrm{kA}$, beta function is $10 \mathrm{~m}$. Right plot: beam current is $1 \mathrm{kA}$, beta function is $5 \mathrm{~m}$.
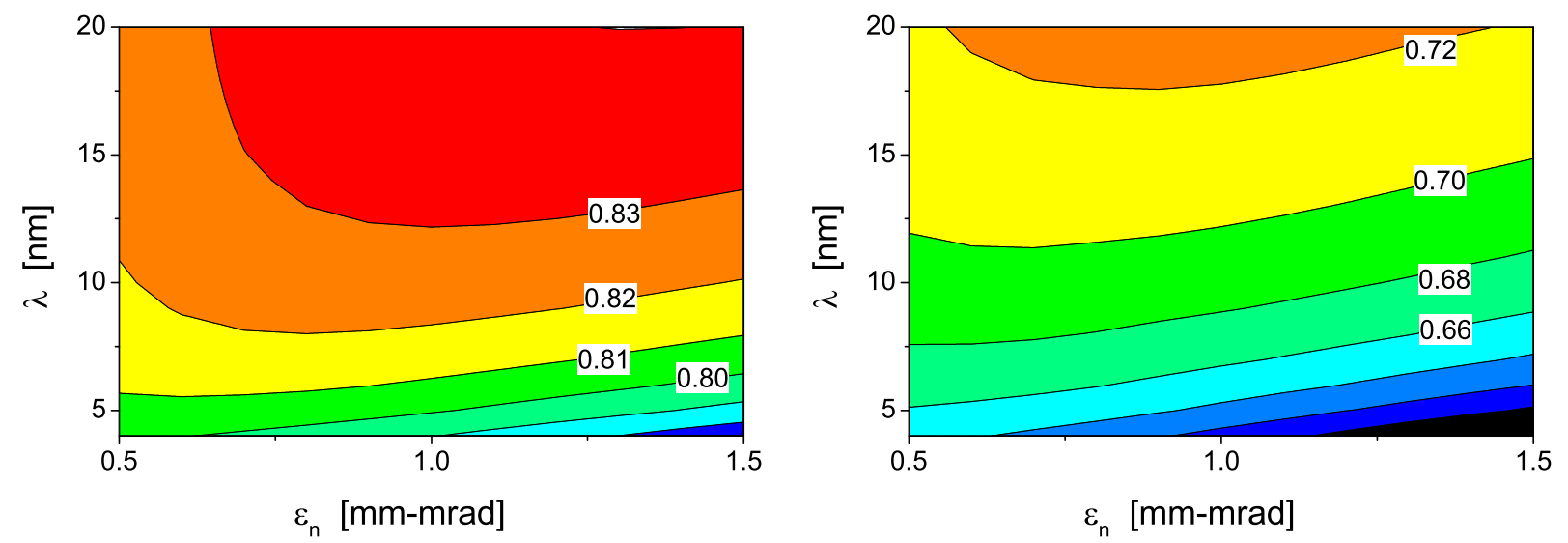

Figure 6. Contour plot of the ratio of the maximum field gain of $\mathrm{TEM}_{10}$ to the field gain of the ground TEM $\mathrm{T}_{00}$ mode versus radiation wavelength and emittance. Left plot: beam current is $1.5 \mathrm{kA}$, beta function is $10 \mathrm{~m}$. Right plot: beam current is 1 $\mathrm{kA}$, beta function is $5 \mathrm{~m}$.

are: the diffraction parameter is $B=17.2$, the energy spread parameter $\hat{\Lambda}_{\mathrm{T}}^{2}=1.7 \times 10^{-3}$, betatron motion parameter $\hat{k}_{\beta}=5.3 \times 10^{-2}$.

Then the reduced parameters at other working points can be easily recalculated using the scaling:

$$
\hat{k}_{\beta} \propto \frac{1}{\beta I^{1 / 2} \lambda^{1 / 4}} \quad \hat{\Lambda}_{\mathrm{T}}^{2} \propto I \lambda^{1 / 2} \quad B \propto \frac{\epsilon_{n} \beta I^{1 / 2}}{\lambda^{1 / 4}} .
$$

The effective contribution of the emittance to the longitudinal velocity spread (4) scales as follows

$$
\left(\hat{\Lambda}_{\mathrm{T}}^{2}\right)_{e f f} \propto \frac{\epsilon_{n}^{2}}{\beta^{2} I \lambda^{3 / 2}}
$$

and equals $2.3 \times 10^{-3}$ at the considered working point.

Analyzing these simple dependencies in terms of their effect on mode separation, we can state that

- Dependencies on the wavelength are relatively weak (except for $\left(\hat{\Lambda}_{\mathrm{T}}^{2}\right)_{\text {eff }}$ ), i.e. one should not expect a significantly better transverse coherence at longer wavelengths. Moreover, mode separation can even be somewhat improved at shorter wavelengths due to a significant increase 
in $\left(\hat{\Lambda}_{\mathrm{T}}^{2}\right)_{e f f}$.

- Reduction of the peak current (by going to a weaker bunch compression) would lead to an improvement of mode separation (even though the energy spread parameter would smaller). Obviously, the peak power at the saturation would be reduced.

- Dependence on the normalized emittance is expected to be weak because of the two competing effects. Mode separation due to a change of the diffraction parameter can be to a large extent compensated by a change of the longitudinal velocity spread. As we will see below, this happens indeed in the considered parameter range.

- Reduction of the beta-function would be the most favorable change because it would reduce the diffraction parameter, and increase the velocity spread at the same time. Unfortunately, there are technical arguments not supporting such a change in the FLASH undulator. Nevertheless, for illustration we will also present some results for the beta-function equal to 5 m.

Contour plot for the value of the diffraction parameter $B$ for the value of beta function of $10 \mathrm{~m}$ and the value of beam current $1.5 \mathrm{kA}$ is presented in Fig. 5 . We see that the value of the diffraction parameter is $B \gtrsim 10$ in the whole parameter space. Figure 6 shows the ratio of the field gain $\operatorname{Re}\left(\Lambda_{10}(\omega)\right)$ to the value of the field gain $\operatorname{Re}\left(\Lambda_{00}(\omega)\right)$ of the fundamental mode. We see that this ratio is above 0.8 in the whole range of parameters, and we can expect significant contribution of the first azimuthal mode to the total radiation power. We can also notice relatively weak dependencies on the emittance and on the wavelength. However, reduction of the current and of the beta-function help to increase the mode separation up to a desirable level as one can see from the right plot in Fig. 6

\section{Simulation procedure}

Simulations have been performed with three-dimensional, time-dependent FEL simulation code [51]. The result of each simulation run contains an array of complex amplitudes $\tilde{E}$ for electromagnetic fields on a three-dimensional mesh. At the next stage of the numerical experiment the data arrays are handled with postprocessor codes to calculate different characteristics of the radiation. Simulations of the statistical properties have been performed for the case of a long bunch with uniform axial profile of the beam current.

The first-order time correlation function $g_{1}\left(t, t^{\prime}\right)$ and transverse correlation function $\gamma_{1}\left(\vec{r}_{\perp}, \vec{r}_{\perp}, z, t\right)$ are defined as

$$
\begin{gathered}
g_{1}\left(\vec{r}, t-t^{\prime}\right)=\frac{\left\langle\tilde{E}(\vec{r}, t) \tilde{E}^{*}\left(\vec{r}, t^{\prime}\right)\right\rangle}{\left[\left\langle|\tilde{E}(\vec{r}, t)|^{2}\right\rangle\left\langle\left|\tilde{E}\left(\vec{r}, t^{\prime}\right)\right|^{2}\right\rangle\right]^{1 / 2}}, \\
\gamma_{1}\left(\vec{r}_{\perp}, \vec{r}_{\perp}, z, t\right)=\frac{\left\langle\tilde{E}\left(\vec{r}_{\perp}, z, t\right) \tilde{E}^{*}\left(\vec{r}_{\perp}, z, t\right)\right\rangle}{\left[\left\langle\left.\tilde{E}\left(\vec{r}_{\perp}, z, t\right)\right|^{2}\right\rangle\left\langle\left|\tilde{E}\left(\vec{r}_{\perp}, z, t\right)\right|^{2}\right\rangle\right]^{1 / 2}},
\end{gathered}
$$

where $\tilde{E}$ is the slowly varying amplitude of the amplified wave. For a stationary random process the coherence time and the degree of transverse are defined as [28]: 


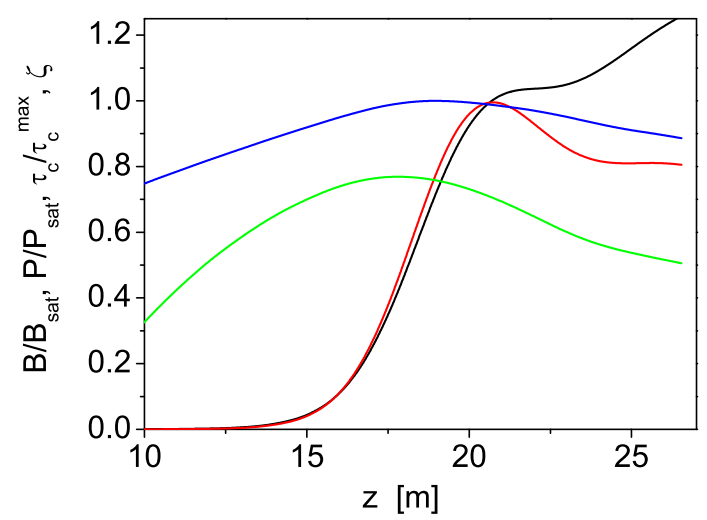

Figure 7. Evolution of the radiation power (black curve), coherence time (blue curve), degree of transverse coherence (green curve), and brilliance (red curve) along the undulator. Brilliance and radiation power are normalized to saturation values. Coherence time is normalized to maximum value of $5.5 \mathrm{fs}$. Radiation wavelength is $8 \mathrm{~nm}$. Beta function is $10 \mathrm{~m}$. Beam current is $1.5 \mathrm{kA}$. rms normalized emittance is $1 \mathrm{~mm}-\mathrm{mrad}$.

$$
\begin{aligned}
\zeta= & \frac{\int\left|\gamma_{1}\left(\vec{r}_{\perp}, \vec{r}_{\perp}\right)\right|^{2} I\left(\vec{r}_{\perp}\right) I\left(\vec{r}_{\perp}\right) \mathrm{d} \vec{r}_{\perp} \mathrm{d} \vec{r}_{\perp}}{\left[\int I\left(\vec{r}_{\perp}\right) \mathrm{d} \vec{r}_{\perp}\right]^{2}} \\
\tau_{\mathrm{c}} & =\int_{-\infty}^{\infty}\left|g_{1}(\tau)\right|^{2} \mathrm{~d} \tau
\end{aligned}
$$

where $I\left(\vec{r}_{\perp}\right)=\left\langle\left|\tilde{E}\left(\vec{r}_{\perp}\right)\right|^{2}\right\rangle$. The first order time correlation function, $g_{1}\left(t, t^{\prime}\right)$, is calculated in accordance with the definition:

Brilliance of the radiation is proportional to the product of the radiation power, coherence time, and of the degree of transverse coherence. Evolution of general characteristics of SASE FEL along the undulator is illustrated in Figure 7. If one traces evolution of the brilliance of the radiation along the undulator length, there is always the point, which we define as the saturation point, where the brilliance reaches maximum value [28].

\section{Results of numerical simulations}

We illustrate general characteristics of FLASH with specific numerical example for FLASH operating at the wavelength of $8 \mathrm{~nm}$, peak current $1.5 \mathrm{kA}$, and rms normalized emittance $1 \mathrm{~mm}-\mathrm{mrad}$.

\subsection{Radiation power}

We present in Figure 8 evolution along the undulator of the radiation power in the fundamental harmonic. Higher values of the peak current and smaller emittances would allow to reach higher radiation powers. When amplification process enters nonlinear stage, the process of nonlinear harmonic generation takes place [45, 52 60]. Figures [10 and 11] show relevant contribution to the total power of the $3 \mathrm{rd}$ and the 5th harmonic. The saturation point and the total undulator length $(27 \mathrm{~m})$ are chosen as reference points. General observation is that the relative contribution of the higher harmonic is higher for smaller values of the emittance. With the value of the normalized emittance of $1 \mathrm{~mm}-\mathrm{mrad}$, partial contributions for the $3 \mathrm{rd}$ and the 5 th harmonic are in the range of $(0.7-1) \times 10^{-2}$ and $(2-2.5) \times 10^{-4}$, respectively. Note that this result is pretty much close to that described by universal scaling with an appropriate correction for longitudinal velocity spread 

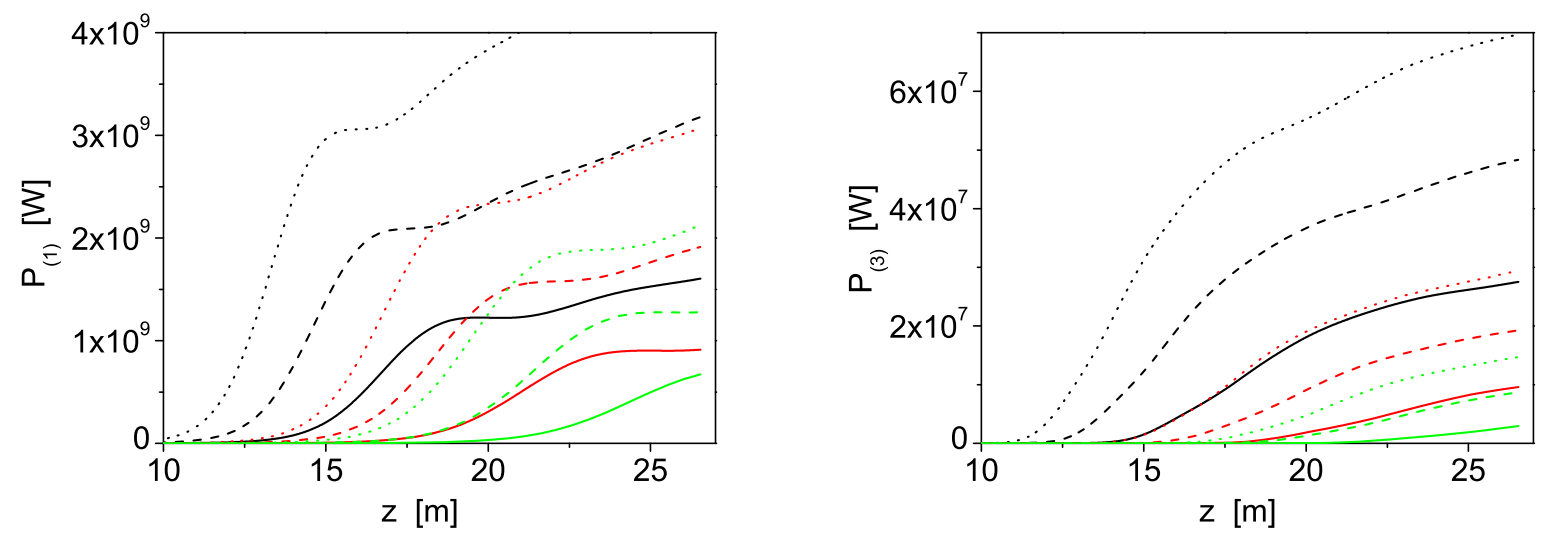

Figure 8. Evolution of the radiation power along undulator for the fundamental harmonic (left plot) and for the 3rd harmonic (right plot). Color codes (black, red and green) refer to different emittance $\epsilon_{n}=0.5,1$, and 1.5 mm-mrad. Line styles (solid, dash, and dot) refer to different values of peak current $1 \mathrm{kA}, 1.5 \mathrm{kA}$, and $2 \mathrm{kA}$. Radiation wavelength is $8 \mathrm{~nm}$. Beta function is $10 \mathrm{~m}$.
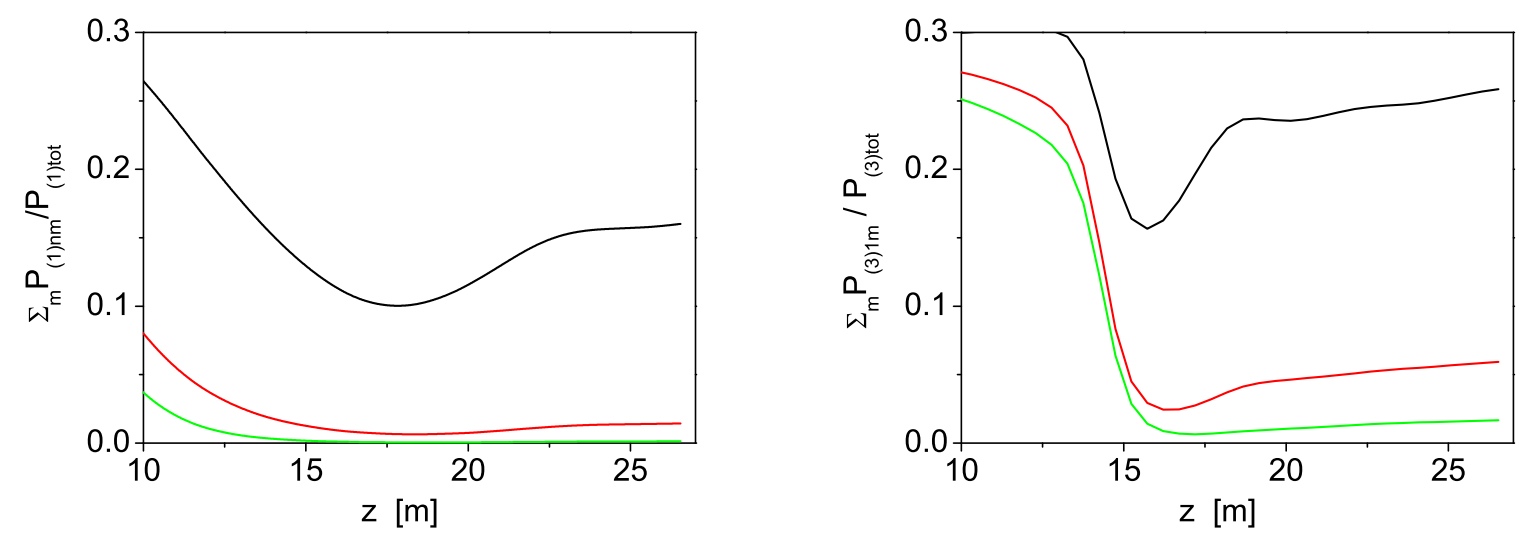

Figure 9. Partial contribution of the higher azimuthal modes for the fundamental harmonic (left plot) and for the 3rd harmonic (right plot). Black, red, and green curves refer to the modes with $n= \pm 1, n= \pm 2$, and $n= \pm 3$, respectively. Radiation wavelength is $8 \mathrm{~nm}$. Beta function is $10 \mathrm{~m}$. Beam current is $1.5 \mathrm{kA}$. rms normalized emittance is $1 \mathrm{~mm}-\mathrm{mrad}$.

derived in 45$]$ :

$$
\left.\frac{\left\langle W_{3}\right\rangle}{\left\langle W_{1}\right\rangle}\right|_{\mathrm{sat}}=0.094 \times \frac{K_{3}^{2}}{K_{1}^{2}},\left.\quad \frac{\left\langle W_{5}\right\rangle}{\left\langle W_{1}\right\rangle}\right|_{\mathrm{sat}}=0.03 \times \frac{K_{5}^{2}}{K_{1}^{2}} .
$$

Here $K_{h}=K(-1)^{(h-1) / 2}\left[J_{(h-1) / 2}(Q)-J_{(h+1) / 2}(Q)\right], Q=K^{2} /\left[2\left(1+K^{2}\right)\right], K$ is rms undulator parameter, and $h$ is an odd integer - harmonic number.

\subsection{Temporal coherence}

In the framework of the one-dimensional model the coherence time at the saturation point is described in terms of FEL parameter $\rho_{1 D}$ [50] and a number of cooperating electrons $N_{\mathrm{c}}=I /\left(e \rho_{1 D} \omega\right)$ [37]:

$$
\tau_{\mathrm{c}} \simeq \frac{1}{\rho_{1 D} \omega} \sqrt{\frac{\pi \ln N_{\mathrm{c}}}{18}}
$$



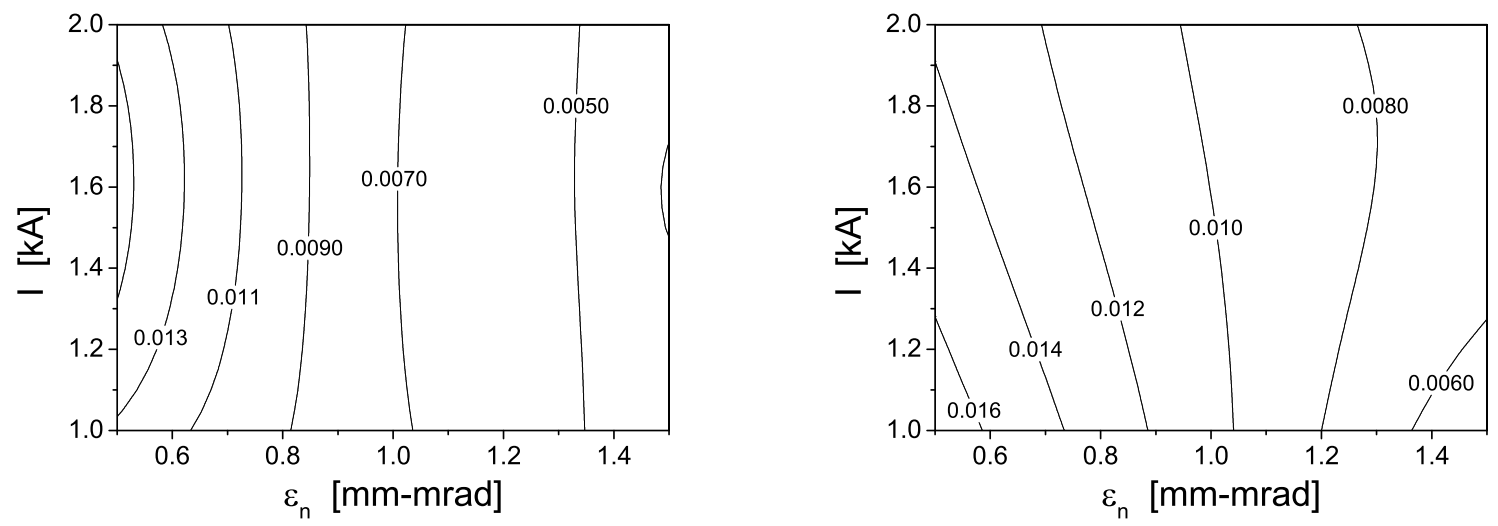

Figure 10. Partial contribution of the 3rd harmonic to the total power versus peak beam current and emittance. Left and right plot refer to the saturation point and the undulator end, respectively. Radiation wavelength is $8 \mathrm{~nm}$. Beta function is 10 $\mathrm{m}$. Beam current is $1.5 \mathrm{kA}$. rms normalized emittance is $1 \mathrm{~mm}-\mathrm{mrad}$.
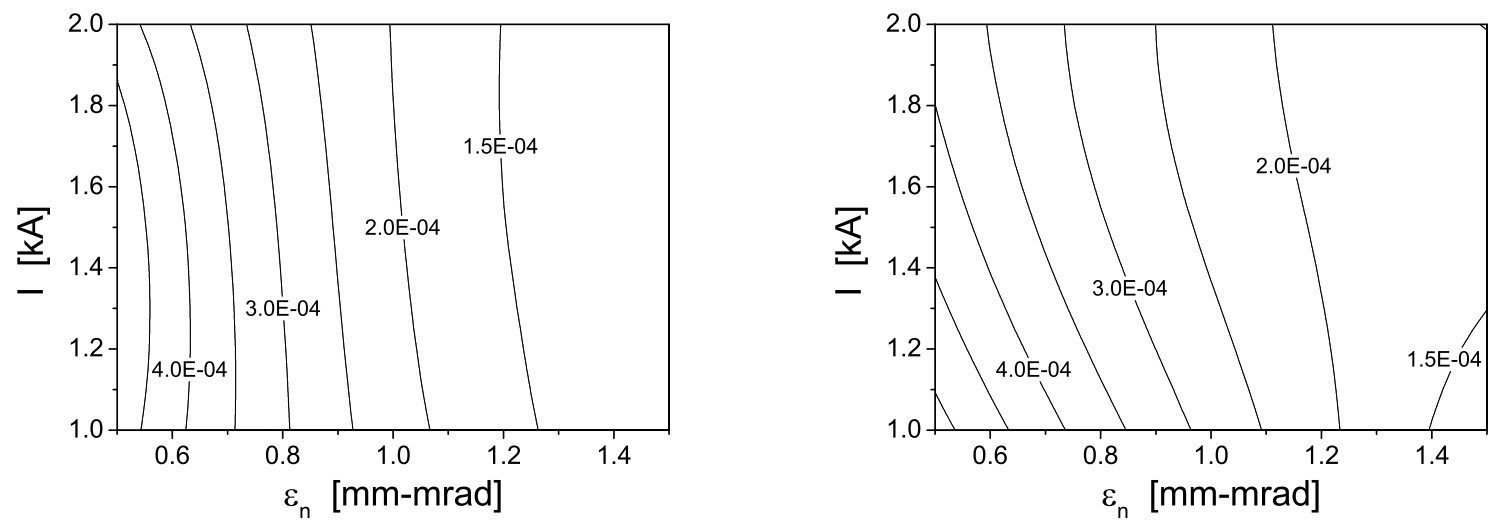

Figure 11. Partial contribution of the 5th harmonic to the total power versus peak beam current and emittance. Left and right plot refer to the saturation point and the undulator end, respectively. Radiation wavelength is $8 \mathrm{~nm}$. Beta function is 10 $\mathrm{m}$. Beam current is $1.5 \mathrm{kA}$. rms normalized emittance is $1 \mathrm{~mm}$-mrad.

The coherence time of higher harmonics at the saturation point and in the post-saturation amplification stage scales inversely proportional to the harmonic number, while relative spectrum bandwidth remains constant with the harmonic number.

Blue curve in Fig. 7 shows evolution of the coherence time which is typical for all SASE FELs. In the high gain linear regime it increases as a square root of undulator length. It reaches maximum value just before saturation point, and then it drops down. Figures 12 show the coherence time for the whole parameter range for the fundamental harmonic. Coherence time for the higher harmonics can be derived using scaling described above.

\subsection{Spatial coherence}

Figure 13 presents an overview of the degree of transverse coherence in the considered parameter space. In our studies of coherent properties of FELs [28] we have found that for an optimized SASE FEL the degree of transverse coherence can be as high as 0.96. One can see from Fig. 13 that in the considered cases the degree of transverse coherence for the 1st harmonic is visibly lower.

We should distinguish two effects limiting the degree of transverse coherence at FLASH. The first one is called mode degeneration and was intensively discussed in this paper. This physical phenomena takes place at large values of the diffraction parameter [37]. Figure9] shows contribution of higher azimuthal modes to the total power for specific example of emittance $1 \mathrm{~mm}$-mrad and 

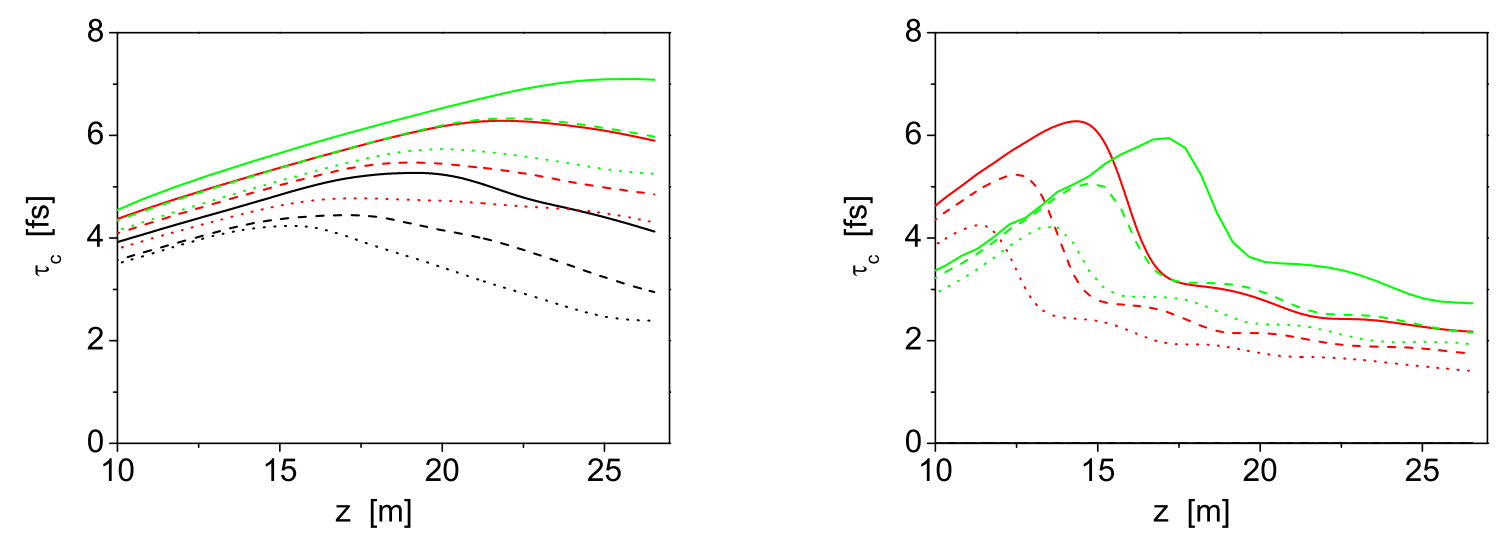

Figure 12. Evolution along undulator of the coherence time of the radiation at the fundamental harmonic (left plot) and at the 3rd harmonic (right plot). Color codes (black, red and green) refer to different emittance $\epsilon_{n}=0.5,1$, and $1.5 \mathrm{~mm}-\mathrm{mrad}$. Line styles (solid, dash, and dot) refer to different values of peak current $1 \mathrm{kA}, 1.5 \mathrm{kA}$, and $2 \mathrm{kA}$. Radiation wavelength is 8 $\mathrm{nm}$. Beta function is $10 \mathrm{~m}$.
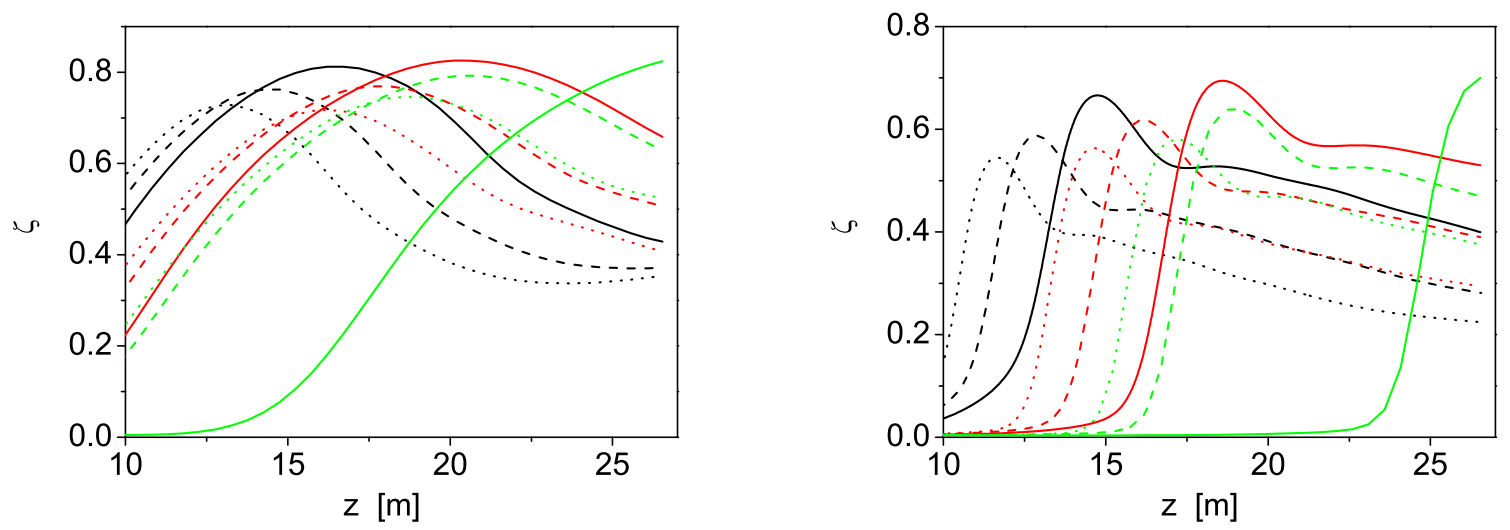

Figure 13. Evolution along the undulator of the degree of transverse coherence of the radiation. Left and right plots correspond to the fundamental frequency $(8 \mathrm{~nm})$ and the $3 \mathrm{rd}$ harmonic $(2.66 \mathrm{~nm})$, respectively. Color codes (black, red and green) refer to different emittance $\epsilon_{n}=0.5,1$, and $1.5 \mathrm{~mm}$-mrad. Line styles (solid, dash, and dot) refer to different values of peak current 1 $\mathrm{kA}, 1.5 \mathrm{kA}$, and $2 \mathrm{kA}$. Radiation wavelength is $8 \mathrm{~nm}$. Beta function is $10 \mathrm{~m}$.

peak current $1.5 \mathrm{kA}$. Contribution of the first azimuthal modes falls down in the high gain linear regime, but to the value of $12 \%$ only, and then starts to grow in the nonlinear regime, and reaches the value of $16 \%$ at the undulator end.

The second effect is connected with a finite longitudinal coherence, it was discovered in [32] and discussed in [28, 29]. The essence of the effect is a superposition of mutually incoherent fields produced by different longitudinally uncorrelated parts of the electron bunch. In the exponential gain regime this effect is relatively weak, but it prevents a SASE FEL from reaching full transverse coherence even in the case when only one transverse eigenmode survives [32]. In the deep nonlinear regime beyond FEL saturation, this effect can be strong and can lead to a significant degradation of the degree of transverse coherence [28, 29]. In particular, as one can see from Fig. 13, this effect limits the degree of transverse coherence to the value about $50 \%$ when FLASH operates in the deep nonlinear regime.

Higher harmonics are derived from the nonlinear process governed by the fundamental harmonic. As a result, coherence properties of the harmonics follow the same tendencies as the fundamental, but with visibly lower degree of transverse coherence [31].

Note that an easiest way to dramatically improve the transverse coherence would be to decrease the beam current such that the saturation is achieved at the very end of the undulator. This would 

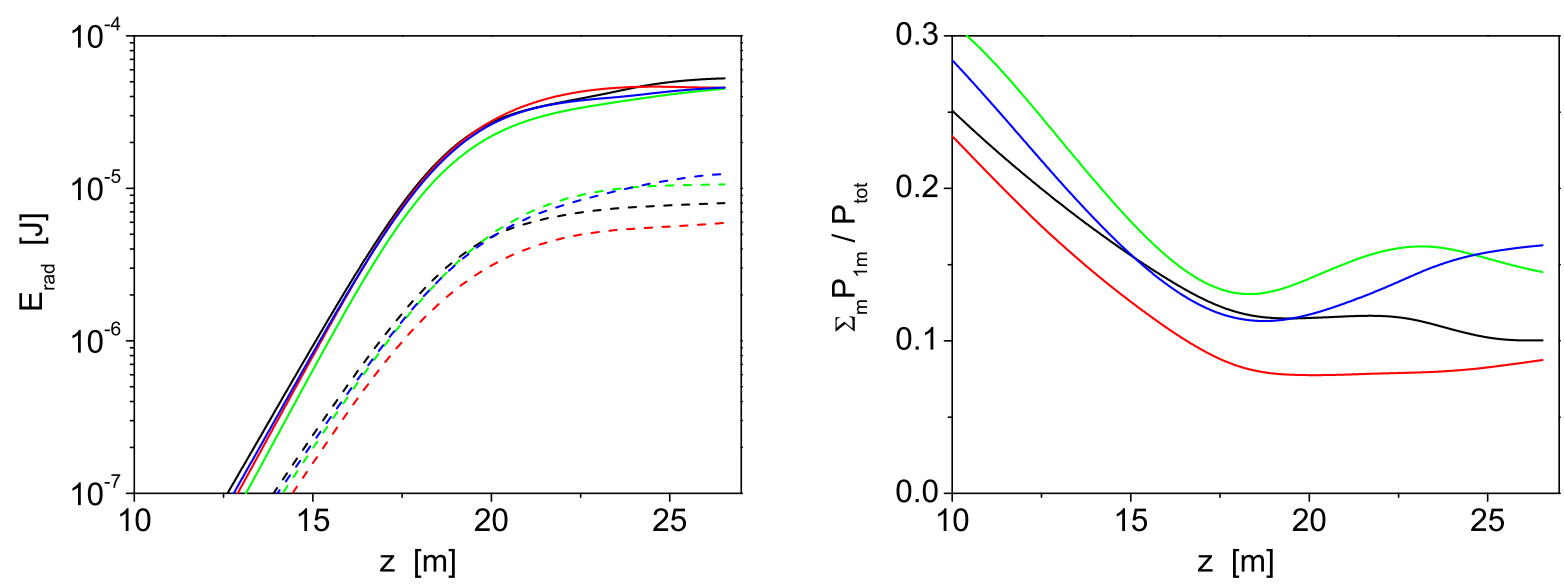

Figure 14. Left plot: evolution of the energy in the radiation pulse versus undulator length. Color codes (black to blue) correspond to different shots. Line style correspond to the total energy in the azimuthally symmetric $\sum T E M_{0 m}$ modes (solid lines), and in of the first azimuthal $\sum T E M_{1 m}$ (dashed lines). Right plot: partial contribution of the first azimuthal modes to the total radiation power, $\sum P_{1 m} / P_{\text {tot }}$. Radiation wavelength is $8 \mathrm{~nm}$. Beta function is $10 \mathrm{~m}$. Beam current is $1.5 \mathrm{kA}$. rms normalized emittance is $1 \mathrm{~mm}$-mrad.

eliminate not only the degradation in the deep nonlinear regime, but would also improve the mode selection process because the diffraction parameter is then reduced while the velocity spread due to emittance is increased. According to our expectations, the degree of transverse coherence might reach the value around $90 \%$ in this regime. Such a regime was realized at FLASH on user's demand, but it is not typical for the machine operation because the peak power is low due to a low peak current.

One can also suppress the unwanted effects in the deep nonlinear regime by kicking the electron beam at the saturation point (or, close to it) when the peak current is high. Then one can still have a high power and an improved (about 70-80\%) degree of transverse coherence. Further improvement could be achieved by reducing beta-function (thus improving the mode selection as discussed above) but this would cause some technical problems that we do not discuss in this paper.

\subsection{Pointing stability and mode degeneration}

Mode degeneration has significant impact on the pointing stability of SASE FEL. Let us illustrate this effect with specific example for FLASH operating with average energy in the radiation pulse of $60 \mu \mathrm{J}$. Left plot in Fig. 14 shows evolution along the undulator of the radiation energy in azimuthally symmetric modes and of the energy in the modes with azimuthal index $n= \pm 1$. Right plot in this figure shows relative contribution to the total radiation energy of the modes with azimuthal index $n= \pm 1$. Four consecutive shots are shown here. Temporal profiles of the radiation pulses are presented in Fig. 15. Intensity distributions in the far zone for these four shots are shown in four rows in Fig. 16. Four profiles on the left-hand side of each row show intensity distributions in the single slices for the time $40 \mathrm{fs}, 50 \mathrm{fs}, 60 \mathrm{fs}$, and $70 \mathrm{fs}$. Right column presents intensity profiles averaged over full shots. We see that transverse intensity patterns in slices have rather complicated shape due to interference of the fields of statistically independent modes with different azimuthal indexes. Shape of the intensity distributions changes on a scale of coherence length. Averaging of slice distributions over radiation pulse results in more smooth distribution. However, it is clearly seen that the spot shape of a short radiation pulse changes from pulse to pulse. The center of gravity of the radiation pulse visibly jumps from shot to shot. Position of the pulse also jumps from shot to shot which is frequently referred as bad pointing stability. Note that the effect illustrated here is a fundamental one which takes place due to the mode degeneration when contribution of the higher azimuthal modes to the total power is pronouncing (10 to 15\% in our case). Only in the case of a long radiation pulse, or after averaging over many pulses we come asymptotically to an azimuthally symmetric radiation distribution (see Fig. 17). Note that 

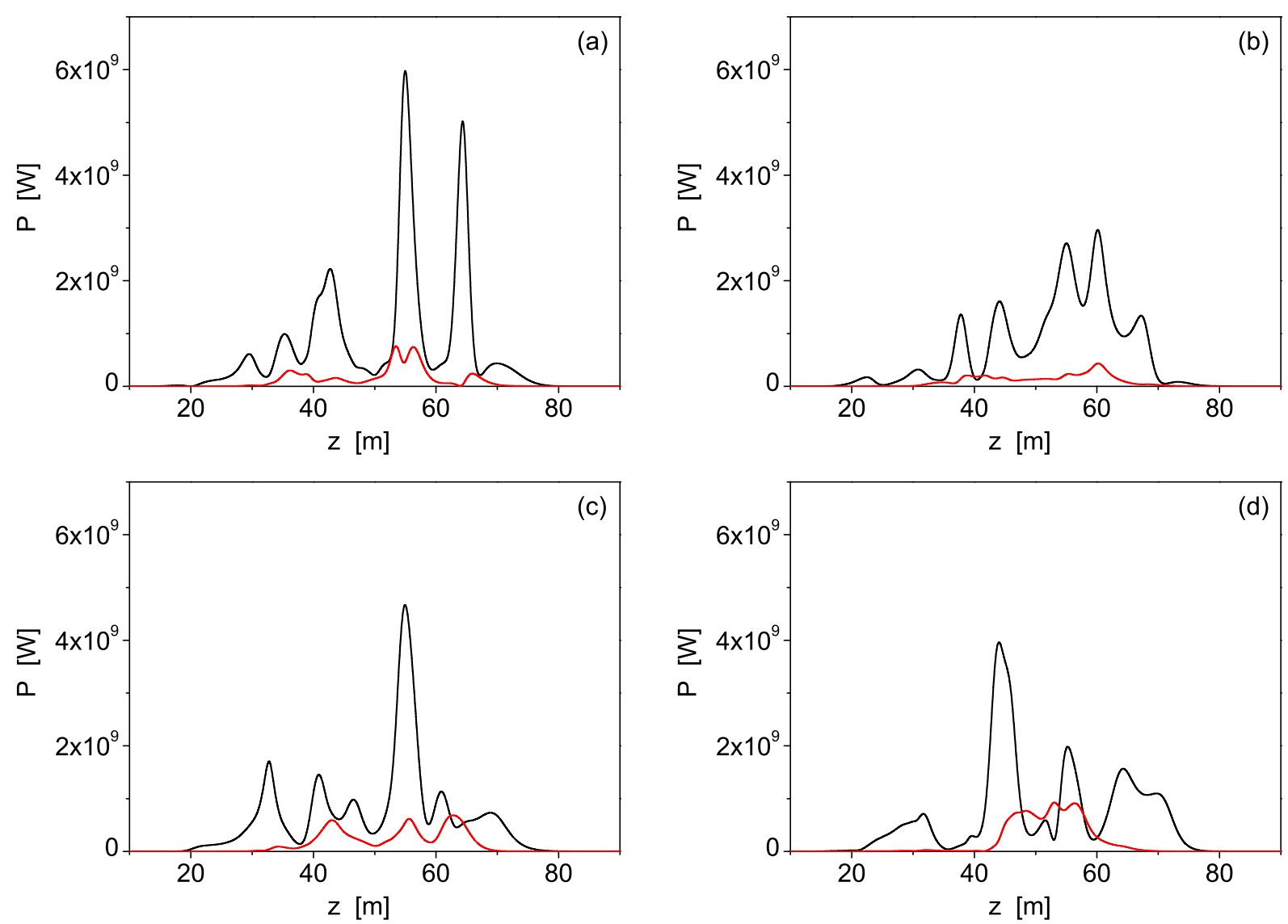

Figure 15. Temporal structure of four radiation pulses. Black lines show the power of the azimuthally symmetric modes, and the curve in the red color show the power of the first azimuthal modes. Radiation wavelength is $8 \mathrm{~nm}$. Beta function is $10 \mathrm{~m}$. Beam current is $1.5 \mathrm{kA}$. rms normalized emittance is $1 \mathrm{~mm}$-mrad. Undulator length is $27 \mathrm{~m}$.

the intensity distributions for the fundamental harmonic are always wider than those for the higher frequency harmonics.

\section{Discussion}

Simulations presented in this paper trace nearly full range of the parameter space of FLASH in terms of the emittance and peak current. Detailed illustration is presented for specific wavelength of $8 \mathrm{~nm}$. We found that there is the fundamental limitation on pointing stability. Also, the degree of transverse coherence at the saturation point is visibly smaller than an ultimate value. This happens because FLASH FEL operates in the range of physical parameters where different radiation modes have close values of the gain, i.e. in the parameter range of mode degeneration. Figure of merit here is the diffraction parameter presenting the ratio of the electron beam size to the diffraction expansion of the radiation on a scale of the field gain length [37]. Power of the effect becomes stronger at the increase of the electron beam size. In the parameter space of FLASH diffraction parameter is in the range between 10 and 30 (see Fig. 5). We have shown that for these values of the diffraction parameter the gain of the first azimuthal mode $T E M_{01}$ approaches to the gain of the ground $T E M_{00}$ mode. The plot in Fig. 6 traces the ratio of the field gain of the first azimuthal mode $T E M_{01}$ to the gain of the ground FEL mode $T E M_{00}$ versus radiation wavelength and emittance. We see that situation with mode selection is unfavorable in the whole wavelength range of FLASH. Ratio is nearly constant which means that detailed results for $8 \mathrm{~nm}$ wavelength can be generalized for the whole parameter space.

The problem of limited transverse coherence has been discussed already at an early stage of the 

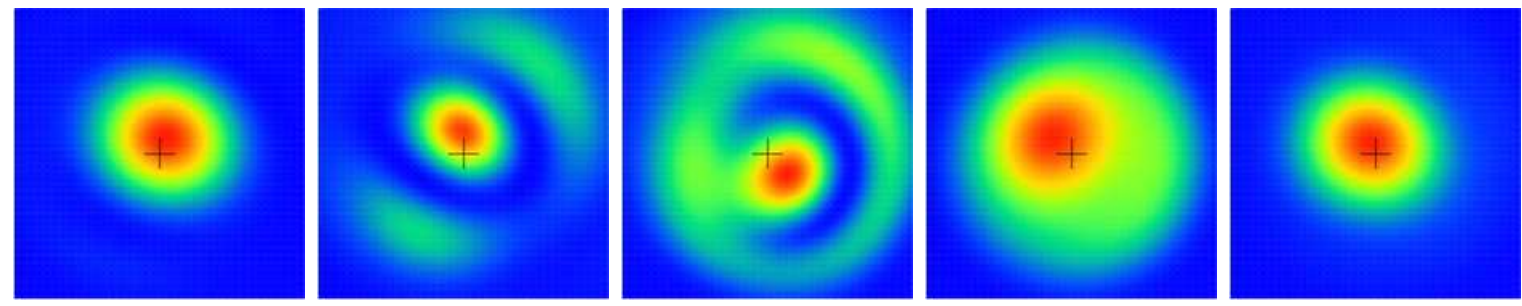

(a)
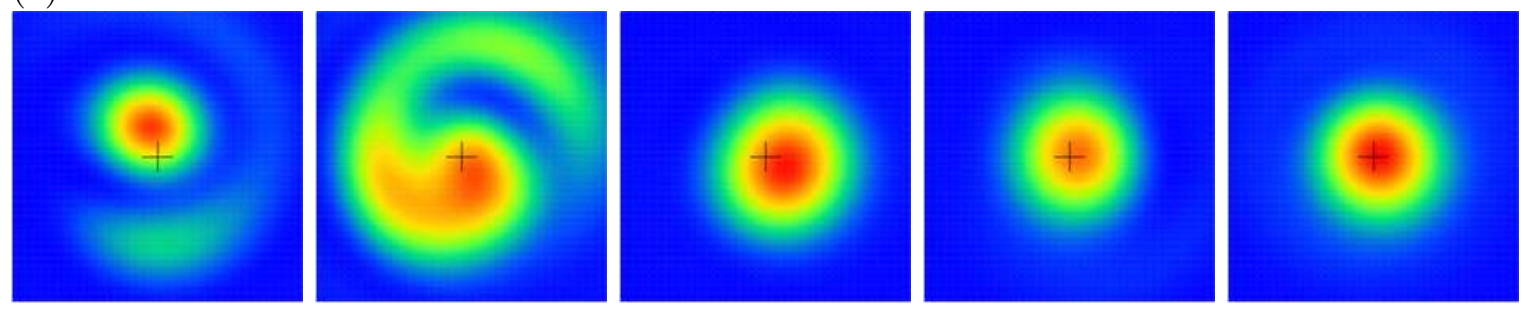

(b)
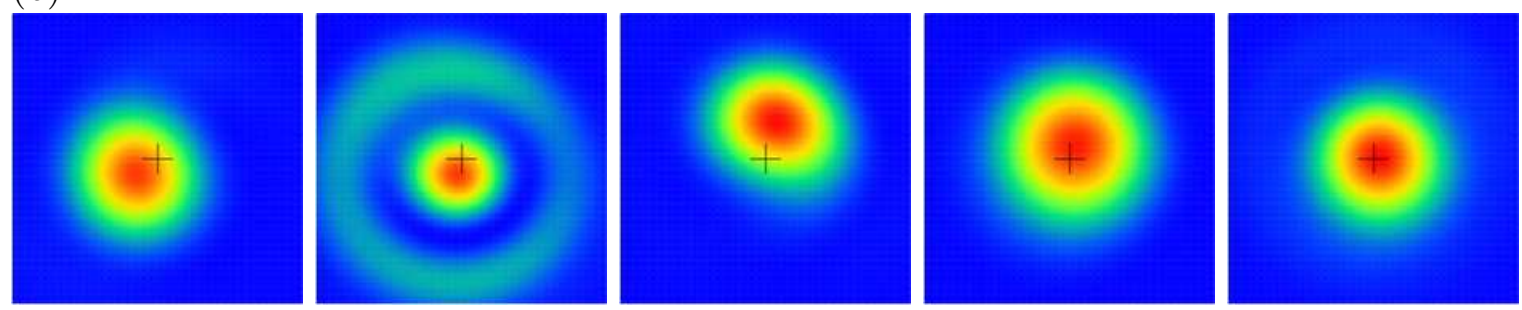

(c)
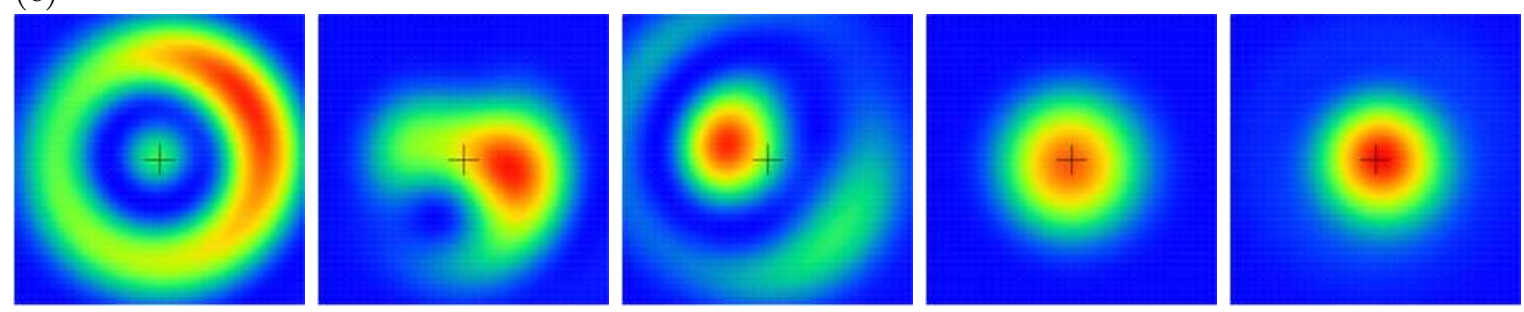

(d)

Figure 16. Profiles of the radiation intensity in the far zone. Rows (a to d) correspond to specific shots with temporal structure presented in Fig. 15 (plots a to d). Profiles on the right-hand side show average intensity over full pulse. Profiles 1 to 4 from the left-hand size show intensity distribution of selected slices corresponding to the time $40 \mathrm{fs}, 50 \mathrm{fs}, 60 \mathrm{fs}$, and $70 \mathrm{fs}$, respectively. Cross denotes geometrical center of the radiation intensity averaged over many shots. Radiation wavelength is $8 \mathrm{~nm}$. Beta function is $10 \mathrm{~m}$. Beam current is $1.5 \mathrm{kA}$. rms normalized emittance is $1 \mathrm{~mm}$-mrad. Undulator length is $27 \mathrm{~m}$.

project [61] which stimulated the design of a strong focusing lattice with superimposed focusing in the undulator [1, 62]. This version of the undulator has been used in the first stage of the project. Present focusing lattice uses quad doublets installed in the undulator intersections, and is capable to provide average beta function down to $5 \mathrm{~m}[63,64]$. Currently FLASH operates with the average focusing function of 10 meters using one quad in the intersection because this allows to improve the reproducibility of the undulator orbit, thus improving dramatically FLASH operability. Otherwise it would be natural to reduce the electron beam size by using the full potential of the focusing lattice with minimum beta function of $5 \mathrm{~m}$. In addition, it would desirable to operate FEL at smaller peak current and lower emittance, say $1 \mathrm{kA}$ and $0.5 \mathrm{~mm}$-mrad. In this case the value of the diffraction parameter goes down to $B=3.5$ for $8 \mathrm{~nm}$ wavelength. This is visibly closer to the diffraction limit. In addition, mode selection is improved due to higher value of the betatron motion parameter $\hat{k}_{\beta}=0.13$. With contour plot in Fig. [6 we can observe the whole range of the parameter space at FLASH for low current, low beta function mode of operation. Numerical simulations confirm the results of the mode analysis. Figure18 shows the contribution of the higher azimuthal modes to the total power for specific example of emittance $0.5 \mathrm{~mm}$-mrad and peak current $1 \mathrm{kA}$. Contribution of the first azimuthal modes falls down to the value of about $1 \%$. Figure 19 demonstrates that 

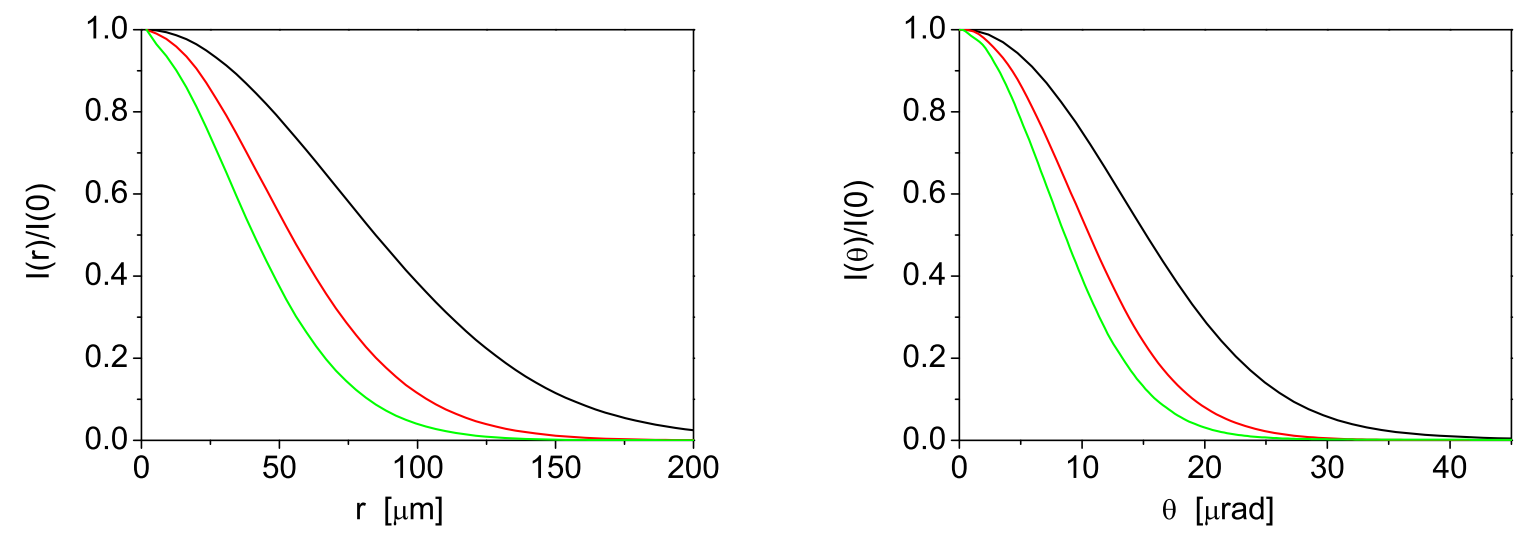

Figure 17. Intensity distribution of the radiation in the saturation point in the near zone (left plot) and far zone (right plot). Black, red, and green curves refer to the 1st, 3rd, and the 5th harmonic, respectively. Radiation wavelength is $8 \mathrm{~nm}$. Beta function is $10 \mathrm{~m}$. Beam current is $1.5 \mathrm{kA}$. rms normalized emittance is $1 \mathrm{~mm}$-mrad.

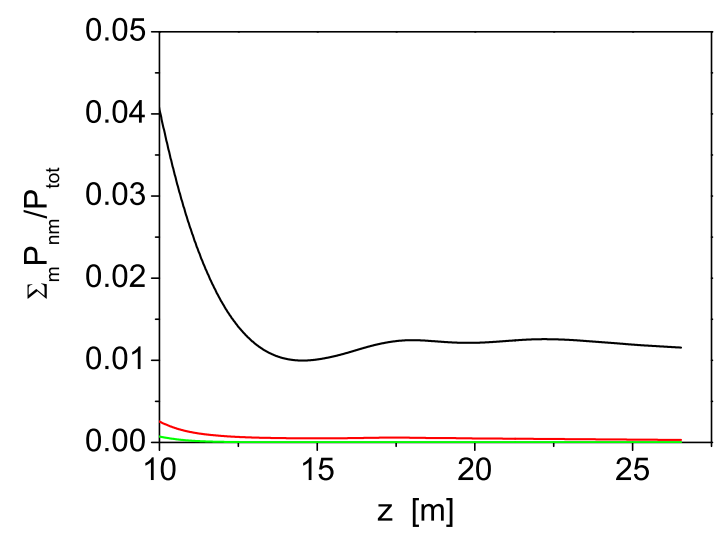

Figure 18. Partial contribution of the higher azimuthal modes of the fundamental harmonic to the total radiation power. Black, red, and green curves refer to the modes with $n= \pm 1, n= \pm 2$, and $n= \pm 3$, respectively. Radiation wavelength is 8 $\mathrm{nm}$. Beta function is $5 \mathrm{~m}$. Beam current is $1 \mathrm{kA}$. rms normalized emittance is $0.5 \mathrm{~mm}$-mrad.

for the fundamental harmonic the maximal degree of the transverse coherence exceeds $90 \%$. The degree of transverse coherence is also high for the 3rd and the 5th harmonic. Note, however, that coherence properties of the radiation degrade significantly when amplification process enters deep nonlinear regime. The physics of this phenomena has been discussed in early papers [28, 29]. Thus, the hint for generating the radiation with the best coherence properties is to tune the machine such that the saturation occurs just in the end of the undulator.

We can also use another mechanism for the suppression of the effect of the mode degeneration. We discussed above that the increase of the energy spread in the electron beam leads to stronger suppression of higher spatial modes. Increase of the energy spread can be done with the laser heater [65]. Features of this effect are demonstrated with Fig. 4. Increase of the rms energy spread to the value of $0.8 \mathrm{MeV}$ in terms of the mode separation is equivalent to the reduction of the beta function from 10 to 5 meters. However, the price for this improvement is significant reduction of the gain of the fundamental mode and of the FEL power, while reduction of the beta function improves these important FEL parameters.

In view of results obtained we conclude that FLASH (and FLASH2) should be operated with as strong focusing of the electron beam as technically possible to provide good spatial coherence and pointing stability of the radiation. Future developments (like design of a new undulator for FLASH) should also take into account these problems and provide relevant technical solutions for keeping small size of the electron beam in the undulator. 


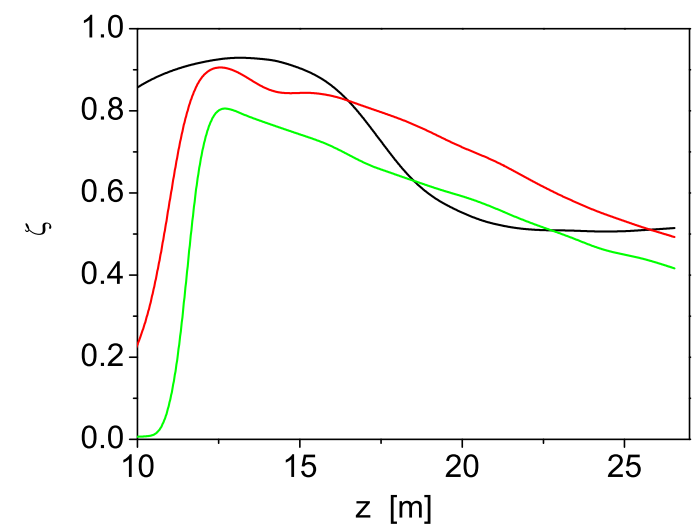

Figure 19. Evolution along undulator of the degree of transverse coherence of the radiation. Color codes (black, red and green) refer to the 1 st harmonic $(8 \mathrm{~nm})$, the 3rd harmonic $(2.66 \mathrm{~nm})$, and the 5 th harmonic $(1.6 \mathrm{~nm})$, respectively. Radiation wavelength is $8 \mathrm{~nm}$. Beta function is $5 \mathrm{~m}$. Beam current is $1 \mathrm{kA}$. rms normalized emittance is $0.5 \mathrm{~mm}$-mrad.

\section{Acknowledgement}

We thank Reinhard Brinkmann for many useful discussions.

\section{References}

[1] TESLA collaboration, D.A. Edwards (ed.), TESLA Test Facility - Design Report, TESLA FEL Report 1995-01, DESY, Hamburg, 1995.

[2] R. Brinkmann, G. Materlik, J. Rossbach, A. Wagner (Eds.), Conceptual Design Report of a $500 \mathrm{GeV}$ e+e- Linear Collider with Integrated X-ray Laser Facility, Preprint DESY 1997-048, ECFA 1997-182, DESY, Hamburg, 1997.

[3] F. Richard, J.R. Schneider. D. trines, A. Wagner (Eds.)., TESLA Technical Design Report. Preprint DESY-2001-011, ECFA-2001-209, TESLA-2001-23, TESLA-FEL-2001-05.

[4] T. Aberg et al., A VUV Free Electron Laser at the TESLA Test Facility at DESY - Conceptual Design Report, TESLA FEL Report 1995-03, DESY, Hamburg, 1995.

[5] J. Andruszkow et al., Phys. Rev. Lett. 85, 3825 (2000).

[6] V. Ayvazyan et al., the femtosecond regime. Phys. Rev. Lett. 88, 104802 (2002).

[7] V. Ayvazyan et al., Eur. Phys. J. D 20, 149 (2002).

[8] H. Wabnitz et al., Nature 420, 482 (2002).

[9] L. Juha et al., Nucl. Instrum. and Methods A 507, 577 (2003).

[10] V. Ayvazyan et al., Eur. Phys. J. D 37, 297 (2006).

[11] W. Ackermann et al., Nature Photonics 1(2007)336

[12] S. Schreiber et al., Proc. of FEL 2012 Conference,http://accelconf.web.cern.ch/AccelConf/FEL2012/papers/ mopd01.pdf

[13] M. Vogt et al., Proc. IPAC 2013 Conference, http://accelconf.web.cern.ch/AccelConf/IPAC2013/papers/ tupea004.pdf.

[14] K. Honkavaara et al., Proc. FEL2013 Conference, New York, USA, 2013, wepso26. http://accelconf.web.cern.ch/AccelConf/FEL2013/papers/weps026.pdf

[15] M. Vogt et al. Proc. IPAC 2014 Conf., paper TUOCA02 (2014), http://accelconf.web.cern.ch/AccelConf/IPAC2014/papers/tupca02.pdf

[16] K. Honkavaara et al., Proc. FEL2014 Conference, Basel, Switzerland, 2014, web05.

[17] R. Brinkmann et al. (eds.), TESLA XFEL. First Stage of the X-Ray Laser Laboratory - Technical Design Report - Supplement. Preprint DESY 2002-167, DESY, Hamburg, 2002.

[18] M. Altarelli et al. (Eds.), XFEL: The European X-Ray Free-Electron Laser. Technical Design Report, Preprint DESY 2006-097, DESY, Hamburg, 2006 (see also http://xfel.desy.de).

[19] Ties Benke et al. (Eds.), The International Linear Collider - Technical Design Report, 2013. http://www.linearcollider.org/ILC/Publications/Technical-Design-Report 
[20] H. Weise, Proc. IPAC2014 Conference, weib03, http://accelconf.web.cern.ch/AccelConf/IPAC2014/papers/weib03.pdf

[21] Phys. Rev. B 79, 212406 (2009).

[22] A.P. Mancuso et al., Phys. Rev. Lett. 102, 035502 (2009).

[23] A. Singer et al, Optics Express, Vol. 20, Issue 16, pp. 17480-17495 (2012)

[24] A.M. Kondratenko, E.L. Saldin, Accelerators 10, 207 (1980).

[25] Y.S. Derbenev, A.M. Kondratenko, E.L. Saldin, Nucl. Instrum. Methods 193, 415 (1982).

[26] J.B. Murphy, C. Pellegrini, Nucl. Instrum. Methods A 237, 159 (1985.)

[27] R. Bonifacio, F. Casagrande and L. De Salvo Souza, Phys. Rev. A 33, 2836 (1986).

[28] E.L. Saldin, E.A. Schneidmiller, and M.V. Yurkov, Opt. Commun. 281(2008)1179.

[29] E.L. Saldin, E.A. Schneidmiller, and M.V. Yurkov, New J. Phys. 12 (2010) 035010, doi: 10.1088/13672630/12/3/035010.

[30] E.L. Saldin, E.A. Schneidmiller, and M.V. Yurkov, Opt. Commun. 281(2008)4727.

[31] E.A. Schneidmiller, and M.V. Yurkov, Proc. FEL 2012 Conference, http://accelconf.web.cern.ch/AccelConf/FEL2012/papers/mopd08.pdf.

[32] E.L. Saldin, E.A. Schneidmiller, and M.V. Yurkov, Opt. Commun. 186(2000)185.

[33] G. Moore, Opt. Commun. 52, 46 (1984).

[34] E.L. Saldin, E.A. Schneidmiller and M.V. Yurkov, Optics Commun 97(1994)272.

[35] M. Xie, Nucl. Instrum. and Methods A 445, 59 (2000).

[36] E.L. Saldin, E.A. Schneidmiller and M.V. Yurkov, Nucl. Instrum. and Methods A 475, 86 (2001).

[37] E.L. Saldin, E.A. Schneidmiller, M.V. Yurkov, "The Physics of Free Electron Lasers" (Springer-Verlag, Berlin, 1999).

[38] K.J. Kim, Phys. Rev. Lett. 57(1986)1871.

[39] J.M. Wang and L.H. Yu, Nucl. Instrum. and Methods A250(1986)484.

[40] S. Krinsky and L.H. Yu, Phys. Rev. A 35(1987)3406.

[41] R. Bonifacio et al., Nucl. Instrum. and Methods A341(1994)181.

[42] R. Bonifacio et al., Phys. Rev. Lett. 73(1994)70.

[43] E.L. Saldin, E.A. Schneidmiller and M.V. Yurkov, Opt. Commun. 148(1998)383.

[44] K.J. Kim, Phys. Rev. Lett. 57(1986)1871.

[45] E.L. Saldin, E.A. Schneidmiller and M.V. Yurkov, Phys. Rev. ST-AB 9(2006)030702

[46] P. Emma et al. Nature Photonics 4, 641 (2010).

[47] T. Ishikawa et al., Nature Photonics 6, 540 (2012).

[48] R. Ganter (Ed.), Swiss FEL Conceptual Design Report, PSI Bericht Nr. 10-04, April 2012.

[49] H.S. Kang, K.W. Kim, I.S. Ko, Proc. IPAC 2014 Conf., paper THPRO019 (2014), http://accelconf.web.cern.ch/AccelConf/IPAC2014/papers/thpro019.pdf

[50] R. Bonifacio, C. Pellegrini and L.M. Narducci, Opt. Commun. 50 (1984) 373.

[51] E.L. Saldin, E.A. Schneidmiller, and M.V. Yurkov, Nucl. Instrum. and Methods A 429(1999)233.

[52] M. Schmitt and C. Elliot, Phys. Rev. A, 34(1986)6.

[53] R. Bonifacio, L. De Salvo, and P. Pierini, Nucl. Instr. Meth. A 293(1990)627.

[54] W.M. Fawley, Proc. IEEE Part. Acc. Conf., 1995, p.219.

[55] H. Freund, S. Biedron and S. Milton, Nucl. Instr. Meth. A 445(2000)53.

[56] H. Freund, S. Biedron and S. Milton, IEEE J. Quant. Electr. 36(2000)275.

[57] S. Biedron et al., Nucl. Instr. Meth. A 483(2002)94.

[58] S. Biedron et al., Phys. Rev. ST 5(2002)030701.

[59] Z. Huang and K. Kim, Phys. Rev. E, 62(2000)7295.

[60] Z. Huang and K. Kim, Nucl. Instr. Meth. A 475(2001)112.

[61] E.L. Saldin, E.A. Schneidmiller, and M.V. Yurkov, TESLA FEL Report 1995-02, DESY, Hamburg, 1995.

[62] Yu. M. Nikitina and J. Pflueger, Nucl. Instrum. and Methods A 381, 554 (1996).

[63] B. Faatz and J. Pflueger, Nucl. Instrum. and Methods A 475, 603 (2001).

[64] J. Pflueger et al., Nucl. Instrum. and Methods A 507, 228 (2002).

[65] E.L. Saldin, E.A. Schneidmiller, and M.V. Yurkov, Nucl. Instrum. and Methods A 528, 355 (2004). 


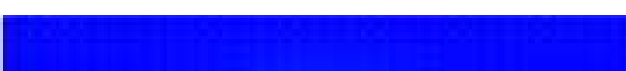

